\title{
Germinação de sementes da invasora Sesbania virgata (Cav.) Pers. sob efeito de luz, temperatura e superação de dormência
}

\author{
Germination of seeds of the invasive plant Sesbania virgata (Cav.) \\ Pers. under effects of light, temperature, and dormancy overcoming
}

\author{
Vênia Camelo de Souza ${ }^{1}$; Pollyanna Freire Montenegro Agra ${ }^{2}$; Leonaldo Alves de \\ Andrade $^{3 *}$; Irenice Gomes de Oliveira ${ }^{2}$; Lamartine Soares de Oliveira ${ }^{4}$
}

\begin{abstract}
Resumo
Este trabalho teve como objetivos avaliar a influência da luz, da temperatura e da escarificação química sobre a germinação de sementes de $S$. virgata, espécie ainda pouco estudada e que se constata invasora em diversos nichos do bioma Caatinga. Sementes de $S$. virgata foram coletadas no município de NatubaPB em março de 2008 e submetidas a oito tratamentos, descritos a seguir: sementes intactas, na presença e ausência de luz, a $25^{\circ} \mathrm{C}$; sementes intactas na presença e ausência de luz, a $30^{\circ} \mathrm{C}$; sementes imersas em ácido sulfúrico por cinco minutos na presença e ausência de luz, a $25^{\circ} \mathrm{C}$; sementes imersas em ácido sulfúrico por cinco minutos na presença e ausência de luz, a $30^{\circ} \mathrm{C}$. Para estimar os efeitos dos tratamentos foram realizados testes de germinação em câmaras de germinação sob luz e escuro contínuo a temperaturas constantes de $25^{\circ} \mathrm{C}$ e $30^{\circ} \mathrm{C}$, em fotoperíodo de 12 horas. As sementes foram colocadas para germinar em caixas Gerbox forradas com folha de papel de filtro. Constatou-se que as sementes de $S$. virgata possuem dormência tegumentar e tem baixa porcentagem de germinação quando intactas, o que constitui uma estratégia para formação de bancos de sementes. A escarificação ácida provocou aumento da germinação e da velocidade de germinação das sementes.
\end{abstract}

Palavras-chave: Invasão biológica, espécie exótica, caatinga

\begin{abstract}
The work had as objectives to evaluate the influence of the light, temperature, and chemical scarification on the germination of seeds of $S$. virgata, species still little studied and that is invasive in several niches of the caatinga ecosystem. The seeds of $S$. virgata were collected in the municipal district of NatubaPB in March/2008. Eight treatments were used as follows: intact seeds in the presence and absence of light, at $25^{\circ} \mathrm{C}$; intact seeds in the presence and absence of light, at $30^{\circ} \mathrm{C}$; submerged seeds in sulfuric acid for five minutes in the presence and absence of light, at $25^{\circ} \mathrm{C}$; submerged seeds in sulfuric acid for five minutes in the presence and absence of light, at $30^{\circ} \mathrm{C}$. The germination tests were accomplished in germination cameras under light and continuous darkness at constant temperatures of $25^{\circ} \mathrm{C}$ and $30^{\circ} \mathrm{C}$, in a 12 hours photoperiod. The seeds were put to germinate in plastic boxes (gerbox) with filter paper. The seeds of $S$. virgata have tegument dormancy and show low germination tax when intact, which is a strategy for seed banks formation. The acid scarification increased germination percentage and speed germination index.
\end{abstract}

Key words: Biological invasion, exotic specie, caatinga

\footnotetext{
${ }^{1}$ Bióloga, Doutoranda em Agronomia, CCA - Universidade Federal da Paraíba - UFPB, Professora do Departamento de Ciências Básicas e Sociais, CCHSA/UFPB, Bananeiras - PB; E-mail: venia@cft.ufpb.br

${ }^{2}$ Mestranda em Agronomia, CCA/UFPB, Areia-PB; E-mail:polly_montenegro@hotmail.com; nicecrat@hotmail.com

${ }^{3}$ Professor Associado do Departamento de Fitotecnia - Laboratório de Ecologia Vegetal, CEP: 58397- 000, Areia, PB; E-mail: landrade@cca.ufpb.br

${ }^{4}$ Mestrando em Ciência Florestal, Universidade Federal de Pernambuco, Recife-PE; E-mail: soareslt@hotmail.com.

* Autor para correspondência
} 
A espécie Sesbania virgata (Cav.) Pers., família Fabaceae-Faboiedeae, possui porte arbustivo e é um taxón pioneiro e semi-perene. $\mathrm{Na}$ região Nordeste a espécie era praticamente desconhecida a cerca de duas décadas, quando então começou a ocupar margens de rios e reservatórios, revelandose mais recentemente como uma invasora capaz de provocar sérios impactos nos ecossistemas invadidos (ANDRADE, 2006). De acordo com o mesmo autor, $S$. virgata se apresenta como invasora na caatinga e ecossistemas associados, particularmente em matas ciliares, onde é capaz de suprimir a regeneração natural das espécies vegetais nativas e formar maciços populacionais dominantes. Andrade, Fabricante e Alves (2008), ao estudarem os impactos da invasora Prosopis juliflora na caatinga constataram redução significativa do número de espécies autóctones, da abundância de indivíduos, assim como da viabilidade das populações nativas, o que já pode estar acontecendo também nas áreas invadidas por Sesbania virgata.

Algumas características permitem que as espécies exóticas se tornem potenciais invasoras: alta taxa de crescimento relativo, grande produção de sementes pequenas e de fácil dispersão, alta longevidade das sementes no solo, alta taxa de germinação dessas sementes, maturação precoce das plantas já estabelecidas, floração e frutificação mais prolongadas, alto potencial reprodutivo por brotação, pioneirismo, alelopatia e ausência de inimigos naturais (GENOVESI, 2005; PARKER et al., 1999).

As sementes de $S$. virgata apresentam dormência tegumentar, o que garante a sobrevivência das mesmas até que estas encontrem condições favoráveis para se desenvolver (ROBERTS, 1981), propiciando assim mais uma vantagem competitiva ao referido táxon.

Luz e temperatura são considerados os dois principais fatores ambientais no controle da germinação (BENECH-ARNOLD; SÁNCHEZ 1995), além da umidade. Em geral, a luz é necessária para a germinação de sementes pequenas (ROSA; FERREIRA, 2001), cujas espécies estão associadas a ambientes abertos. A relação entre germinação e temperatura está frequentemente associada às temperaturas a que as plantas ficam expostas durante a fase de crescimento (VILLALOBOS; PELÁEZ, 2001 apud OLIVEIRA; GARCIA, 2005). Em algumas espécies o requerimento de luz para germinação das sementes é fortemente influenciado pela temperatura (SMITH, 1975), e a faixa de variação de temperatura na qual as sementes podem germinar é uma característica de cada espécie (BEWLEY; BLACK, 1994). Os limites extremos de temperatura para germinação fornecem informações de interesse ecológico (LABOURIAU; PACHECO, 1978), sendo importante a determinação das temperaturas mínima, ótima e máxima para cada espécie. A temperatura ótima propicia a máxima porcentagem de germinação em menor espaço de tempo, enquanto temperaturas acima da máxima e abaixo da mínima as sementes pouco germinam (BEWLEY; BLACK, 1994).

Pelo exposto, estudos que abordam aspectos ecofisiológicos da germinação são essenciais para o entendimento do sucesso do estabelecimento das espécies em campo, que são determinados pela faixa de condições ambientais toleradas pelas sementes durante a germinação (MALUF; MARTINS, 1991), principalmente quando se trata de uma espécie invasora. Assim, o presente trabalho teve como objetivos avaliar a influência da luz, da temperatura e da escarificação química sobre a germinação de sementes de $S$. virgata, espécie ainda pouco estudada e que se constata invasora em diversos habitats do bioma caatinga.

O experimento foi instalado no Laboratório de Ecologia Vegetal, do Centro de Ciências Agrárias, Universidade Federal da Paraíba, no período de junho a agosto de 2008. As sementes de $S$. virgata utilizadas foram coletadas em uma área de mata ciliar do Rio Paraíba, no município de Natuba-PB, em março de 2008. Foram utilizados oito tratamentos, em esquema fatorial 2x2x2 (duas condições de luz, 
duas temperaturas e duas condições de quebra de dormência) descritos a seguir:1) sementes intactas na presença de luz, a $25^{\circ} \mathrm{C}$; 2) sementes intactas na ausência de luz, a $25^{\circ} \mathrm{C} ; 3$ ) sementes intactas na presença de luz, a $30^{\circ} \mathrm{C}$; 4) Sementes intactas na ausência de luz, a $30^{\circ} \mathrm{C}$; 5) sementes imersas em ácido sulfúrico por 5 minutos na presença de luz, a $25^{\circ} \mathrm{C}$; 6) sementes imersas em ácido sulfúrico por 5 minutos na ausência de luz, a $25^{\circ} \mathrm{C}$; 7) sementes imersas em ácido sulfúrico por 5 minutos na presença de luz, a $30^{\circ} \mathrm{C}$; 8) sementes imersas em ácido sulfúrico por 5 minutos na ausência de luz, a $30^{\circ} \mathrm{C}$. Os testes de germinação foram realizados em câmaras de germinação sob luz e escuro contínuo a temperaturas constantes de $25^{\circ} \mathrm{C}$ e $30^{\circ}$ $\mathrm{C}$, em fotoperíodo de 12 horas. As sementes foram colocadas para germinar em caixas Gerbox forradas com folha de papel de filtro umedecido com 2,5 vezes o peso do papel. A simulação da condição de escuro contínuo foi realizada envolvendo-se as caixas Gerbox em papel alumínio e em sacos pretos de polietileno, sendo o acompanhamento feito em câmara escura, com luz verde de segurança. Para todos os tratamentos foram utilizadas 100 sementes, distribuídas em 4 repetições de 25 sementes. $\mathrm{O}$ delineamento experimental utilizado foi o inteiramente casualizado. A germinação foi avaliada diariamente, até a estabilização da resposta. A velocidade de germinação (IVG) foi calculada segundo Labouriau (1970). Os dados, não transformados, foram submetidos à análise de variância pelo teste $\mathrm{F}$ e as médias comparadas pelo teste de Tukey, a $5 \%$ de probabilidade.

A luz não influenciou a germinação de sementes de S. virgata, por esse motivo os tratamentos com e sem luz foram agrupados na apresentação dos resultados (Tabela 1). A ausência de influência da luz revela mais uma estratégia da espécie para ocupar outros nichos ou formar populações adensadas, uma vez que seus propágulos podem germinar até mesmo em condições de baixa ou nenhuma luminosidade. Em relação à resposta à luz as sementes são classificadas em três grandes grupos: fotoblásticas positivas (que germinam só ou melhor na luz), fotoblásticas negativas (em que a germinação é inibida pela luz) e as indiferentes (TAKAKI, 2001 apud SANTOS; FERREIRA; ÁQUILA, 2004). A espécie em estudo, de acordo com a classificação citada, se comportou como indiferente. Sementes escarificadas com ácido sulfúrico germinaram em média, nas temperaturas de $25^{\circ}$ e $30^{\circ} \mathrm{C}$, $46,88 \%$ e $57 \%$, respectivamente. Sementes intactas germinaram em média, nas temperaturas de $25^{\circ} \mathrm{C}$ e $30^{\circ} \mathrm{C}, 24,0 \%$ e $11,50 \%$, constatandose, na temperatura de $25^{\circ} \mathrm{C}$, maior porcentual de germinação (Tabela 1). Para o IVG, a escarificação aumentou significativamente a velocidade de germinação $(1,79)$ diferindo estatisticamente das sementes não escarificadas $(0,22)$. Veasey, Freitas e Schammass (2000) estudando a variabilidade da dormência de sementes entre e dentro de cinco espécies de Sesbania, constataram que S. virgata foi a espécie que apresentou menor velocidade de germinação justificando que a germinação mais lenta para esta espécie talvez se deva ao tegumento bastante duro de suas sementes, o qual deve ser inicialmente rompido para que ocorra a germinação da semente.

Roberts (1981), afirma que as sementes de Sesbania virgata apresentam dormência tegumentar, o que garante a sobrevivência da mesma até que esta encontre condições favoráveis para se desenvolver. O tegumento duro e impermeável à entrada de água deve ser o mecanismo responsável para manter a alta longevidade das sementes de Sesbania virgata (BASKIN; BASKIN, 1998 apud VIEIRA; OLIVERIA; SILVEIRA, 2007).

A dormência de sementes é um fator importante na dinâmica de populações naturais, e está relacionada à adaptação dos indivíduos a ambientes heterogêneos. Esta dormência garante que a germinação das sementes e, consequentemente, o desenvolvimento das plântulas, ocorra na época e local mais vantajosos (BASKIN; BASKIN, 1985; BEWLEY;BLACK, 1985 apudVEASEY;FREITAS; SCHAMMASS, 2000). S. virgata pertence ao 
subgênero Daubentonia, que é nativo das Américas (MONTEIRO, 1984), cujas espécies apresentam vagens tetra-aladas e são de grande ocorrência no Estado de São Paulo, geralmente associadas a beira de rios ou brejos e de acordo com Lorenzi, (2000) se reproduz até duas vezes por ano. Andrade (2006) constata que $S$. virgata se apresenta como invasora na caatinga e ecossistemas associados no Nordeste, particularmente em matas ciliares, onde é capaz de suprimir a regeneração natural e formar maciços populacionais dominantes.

Tabela 1.Valores de germinação e Índice de Velocidade de Germinação (IVG) de sementes de Sesbania virgata.

\begin{tabular}{|c|c|c|}
\hline \multicolumn{3}{|c|}{ Germinação (\%) } \\
\hline \multirow[t]{2}{*}{ Escarificação } & \multicolumn{2}{|c|}{ Temperatura } \\
\hline & $25^{\circ} \mathrm{C}$ & $30^{\circ} \mathrm{C}$ \\
\hline Semente intacta & $24,00 \mathrm{~b} \mathrm{~A}$ & $11,50 \mathrm{~b} \mathrm{~B}$ \\
\hline Semente escarificada & 46,88 a A & 57,00 a $\mathrm{A}$ \\
\hline Semente & \multicolumn{2}{|c|}{ IVG } \\
\hline Intacta & \multicolumn{2}{|c|}{$0,22 \mathrm{~b}$} \\
\hline Escarificada & \multicolumn{2}{|c|}{$1,79 \mathrm{a}$} \\
\hline
\end{tabular}

Médias seguidas de mesma não diferem a $5 \%$ de probabilidade pelo teste $\mathrm{F}$

Médias seguidas de mesma letra minúscula na coluna e maiúscula na linha não diferem a 5\% de probabilidade pelo teste $\mathrm{F}$.

A temperatura, na faixa estudada não teve nenhum efeito na velocidade de germinação de sementes de S.virgata. Carpanezzi e Fowler (1997), pesquisando a quebra da dormência tegumentar de sementes de S. virgata constataram também que o ácido sulfúrico concentrado possibilitou maior quebra da dormência tegumentar da espécie. Carvalho e Nakagawa (2000), ressaltaram que a temperatura ótima para germinação total é diferente da ótima, para velocidade de germinação das sementes.

Este trabalho revelou o comportamento da espécie nas condições propostas. As sementes de $S$. virgata apresentam dormência tegumentar, o que representa uma estratégia da espécie para se propagar e ocupar novos nichos frente às variações das condições naturais. Sementes dormentes tendem a apresentar maior longevidade e formar bancos de sementes persistentes no solo o que constitui uma vantagem competitiva, a qual é potencializada significativamente com outras estratégias, a exemplo do rápido crescimento na fase de plântulas.
As sementes de $S$. virgata possuem dormência tegumentar e apresentam baixa taxa de germinação quando intactas, o que constitui uma estratégia para formação de expressivos bancos de sementes.

A escarificação ácida interferiu favoravelmente na germinação e na velocidade de germinação das sementes do referido táxon invasor.

As sementes escarificadas apresentaram comportamento germinativo estatisticamente igual nos dois regimes de temperatura, enquanto as sementes intactas apresentaram germinação superior na temperatura de $25^{\circ} \mathrm{C}$.

Os propágulos do táxon estudado são capazes de germinar em diferentes intensidades luminosas, o que se revela mais uma estratégia de invasão e formação de banco de plântulas até mesmo sob populações adensadas e já estabelecidas. 


\section{Referências}

ANDRADE, L. A. Espécies exóticas invasoras no nordeste do Brasil: impactos nos ecossistemas locais. In: MARIATH, J. E. A.; SANTOS, R. P.(Org). Os avanços da botânica no início do século XXI: morfologia, fisiologia, taxonomia, ecologia e genética. Porto Alegre: Sociedade Botânica do Brasil, 2006, 752 p.

ANDRADE, L. A.; FABRICANTE, J. R.; ALVES, A. S. Algaroba (Prosopis juliflora (Sw)DC.: impactos sobre a fitodiversidade e estratégias de colonização em área invadida na Paraíba. Natureza e Conservação, CuritibaPR, v. 6, n. 2, p. 61-67, 2008.

BASKIN, J. M.; BASKIN, C. C. The annual dormancy cycle in buried weed seeds: a continuum. BioScience, Washington, v. 35, n. 8, p. 492-498, 1985.

BENECH-ARNOLD, R.; SÁNCHEZ, R. A.. Modeling weed seed germination. In. KIGEL, J.; GALILI, G. A. (Ed.). Development e germination. New York: Academic Press. 1995. p. 545-566.

BEWLEY, J. D.; BLACK, M. Seeds: physiology of development and germination. New York: Plenum Press, 1985. $367 \mathrm{p}$.

Seeds: physiology of development and germination. New York: Plenum Press, 1994. 445 p.

CARPANEZZI, A. A.; FOWLER, J. P. A. Quebra da dormência tegumentar de sementes de Sesbania virgata (Cav.) Pers. Colombo: EMBRAPA, 1997, p. 1-2. (Comunicado Técnico, 14).

CARVALHO, N. M.; NAKAGAWA, J. Germinação de sementes. In: Sementes: ciência, tecnologia e produção. 4. ed. Jaboticabal: FUNEP, 2000. p. 128-166.

GENOVESI, P. Eradications of invasive alien species in Europe: a review. Biological Invasions, University of Tennessee, USA, v. 7, p. 127-133, 2005.

LABOURIAU, L. G.; PACHECO, A. On the frequency of isothermal germination in seeds of Dolichos biflorus L. Plant \& CellPhysiology, Oxford:University Press, v. 19, n. 3, p. 507-512, 1978.

LABOURIAU, L. F. G. On the physiology of seed germination in Vicia graminea Sm. In: ANAIS DA ACADEMIA BRASILEIRA DE CIÊNCIAS, 1., 1970, Rio de Janeiro. Anais... Rio de Janeiro, 1970. v. 42. p. 235-262.

LORENZI, H. Plantas daninhas do Brasil: terrestres, aquáticas,parasitas e tóxicas. 3. ed. São Paulo: Nova Odessa, 2000. 69 p.
MALUF, A. M.; MARTINS, P. S. Germinação de sementes de Amaranthus hybridus L. e Amaranthus viridis L. Revista Brasileira de Biologia, Rio de Janeiro, v. 51, n. 3, p. 417-425, 1991.

MONTEIRO, R. Taxonomic studies on Brazilian legumes with forage potential: Sesbania, Lupinus. St. Andrews. Thesis (Ph.D.). University of St. Andrews. 1984. 446 p.

OLIVEIRA, P. G.; GARCIA, Q. S. Efeitos da luz e da temperatura na germinação de sementes de Syngonanthus elegantulus Ruhland, S. elegans (Bong.) Ruhland e S. venustus Silveira (Eriocaulaceae). Acta Botânica Brasílica, São Paulo, v. 19, n. 3, p. 639-645, 2005.

PARKER, I. M.; SIMBERLOFF, D.; LONSDALE, W. M.; GOODELL, K.; WONHAM, M.; KAREIVA, P. M.; WILLIAMSON, M. H.; HOLLE, B. V.; MOYLE, P. B.; BYERS, J. E.; GOLDWASSER, L. Impact: toward a framework for understanding the ecological effects of invaders. Biological Invasions, University of Tennessee, USA, v. 1, p. 3-19. 1999.

ROBERTS, H. A. Seed banks in soils. Advances in Applied Biology, New York, v. 6, n. 1, p. 1-55. 1981.

ROSA, S. G. T.; FERREIRA, A. G. Germinação de plantas medicinais lenhosas. Acta Botanica Brasilica, São Paulo, v. 15, n. 2, p. 147-288, 2001.

SANTOS, C. M. R.; FERREIRA, A. G.; ÁQUILA, M. E. A. Características de frutos e germinação de sementes de seis espécies de Myrtaceae nativas do Rio Grande do Sul. Ciência Florestal, Santa Maria, v. 14, n. 2, p. 13-20. 2004.

SMITH, H. Light quality and germination: ecological implications. In: HEYDECHER, W. Seed ecology. London: Buttrworth, 1975. p. 131-219.

TAKAKI, M. New proposal of classification of seeds based on forms of phytochrome instead of photoblastism. Revista Brasileira de Fisiologia Vegetal, Lavras, v. 13, n. 1, p. 103-107, 2001.

VEASEY, E. A.; FREITAS, J. C. T.; SCHAMMASS, E. A. Variabilidade da dormência de sementes entre e dentro de espécies de Sesbania. Sci. Agric., Piracicaba, v. 57, n. 2, p. 299-304. 2000.

VIEIRA, B. C.; OLIVEIRA, S. M. S.; SILVEIRA, F. A. O. Efeito da luz e escarificação na germinação de sementes de Sesbania virgata (Fabaceae) sob condições artificiais de armazenamento. In: CONGRESSO DE ECOLOGIA DO BRASIL, 8., Caxambu. Anais... Caxambu: Sociedade de Ecologia do Brasil, 2007. p. 1-2. 
